\title{
La educación artística formal y no formal: contextos colaborativos
}

\section{Formal and non-formal arts education: collaborative contexts}

\author{
Magdalena Castejón Ibáñez \\ Universidad de Murcia (España) \\ mmagdalena.castejon@um.es
}

Recibido 28/08/2020 Revisado 10/11/2020

Aceptado 17/11/2020 Publicado 30/11/2020

\section{Resumen:}

El texto expuesto, presenta los resultados de una investigación doctoral finalizada en 2019, en la que se analizaron las posibilidades de actuación del Museo de Bellas Artes de Murcia con respecto a su entorno sociocultural cercano, en cuanto a su potencial como espacio para la educación artística y patrimonial no formal y como catalizador del desarrollo ciudadano. Así mismo, tras una revisión del estudio, se exponen nuevas vías de actuación vinculadas a los resultados del mismo, basadas en la relación entre educación formal y no formal, y las nuevas estrategias de comunicación social.

Sugerencias para citar este artículo,

Castejón Ibáñez, Magdalena (2020). La educación artística formal y no formal: contextos colaborativos. Tercio Creciente (Monográfico extraordinario III), págs. 99-112, https://dx.doi.org/10.17561/rtc.extra3.5715

CASTEJÓN IBÁÑEZ, MAGDALENA (2020) La educación artística formal y no formal: contextos colaborativos. Tercio Creciente (Monográfico extraordinario III), noviembre 2020, pp. 99-112, https://dx.doi.org/10.17561/rtc.extra3.5715 
ISSN: $2340-9096$

https://dx.doi.org/10.17561/rtc.extra3.5715

\section{Abstract:}

The exposed text, shows the results of a doctoral research finished in 2019, in which the possibilities of action of the Museum of Fine Arts of Murcia were analyzed with respect to its close sociocultural environment, in terms of its potential as an artistic-educational space and non-formal heritage, and as a catalyst for citizen development. Likewise, after a review of the study, new ways of action linked to its results are exposed, based on the relation between formal and non-formal education, and new social communication strategies.

Palabras Clave: Museo Museos, educación, participación, colaboración, universidad

Key words: Museum, Education, participation, collaboration, university

\section{Sugerencias para citar este artículo,}

Castejón Ibáñez, Magdalena (2020). La educación artística formal y no formal: contextos colaborativos. Tercio Creciente (Monográfico extraordinario III), págs. 99-112, https://dx.doi.org/10.17561/rtc.extra3.5715

CASTEJÓN IBÁÑEZ, MAGDALENA (2020) La educación artística formal y no formal: contextos colaborativos. Tercio Creciente (Monográfico extraordinario III), noviembre 2020, pp. 99-112, https://dx.doi.org/10.17561/rtc.extra3.5715 


\section{Introducción}

Los museos en la actualidad tienen un cometido crucial más allá de la custodia y exhibición de bienes patrimoniales. Las instituciones museísticas han ido evolucionando hacia una visión mucho más abierta que ha permitido la creación de modelos híbridos caracterizados principalmente por la proximidad a la ciudadanía. Este cambio progresivo está respaldado por la denominada museología social, cuyas bases se asientan a final del siglo XX, y defienden una necesidad de mejorar cualitativamente la relación con la sociedad, haciéndola partícipe de los procesos culturales.

En este texto se presentan los resultados de la investigación culminada en 2019, cuyo objetivo fue plantear una metodología de acercamiento entre museos y ciudadanos. En concreto se escoge como caso específico de estudio el Museo de Bellas Artes de Murcia (MUBAM) (Ilustración 1) y el entorno sociocultural cercano al centro, esto es, los seis barrios limítrofes con el mismo: La Paz, Vistabella, La Fama, San Juan, Santa Eulalia y San Lorenzo.

El cambio paulatino de las instituciones museísticas hacia una relación más estrecha con las comunidades cercanas, debe desarrollarse de manera plural, es decir, teniendo en cuenta las inmensas y diversas posibilidades que ofrece la vinculación y colaboración no solo con los distintos públicos de los museos, sino con los innumerables entes y organizaciones de carácter social, cultural y educativo que componen la sociedad actual.

Pasar del concepto de museo como mero contenedor de colecciones admirables hacia una idea más experimental, en la que tienen cabida nociones como la de laboratorio, no está resultando fácil para todas las instituciones. Son diversas las causas, pero se apunta generalmente a cuestiones económicas. No obstante, tal y como se ha podido demostrar en esta investigación, en muchas ocasiones es tan solo necesario un cambio en las estrategias y metodologías de actuación por parte de la gestión del museo y no tanto un factor monetario.

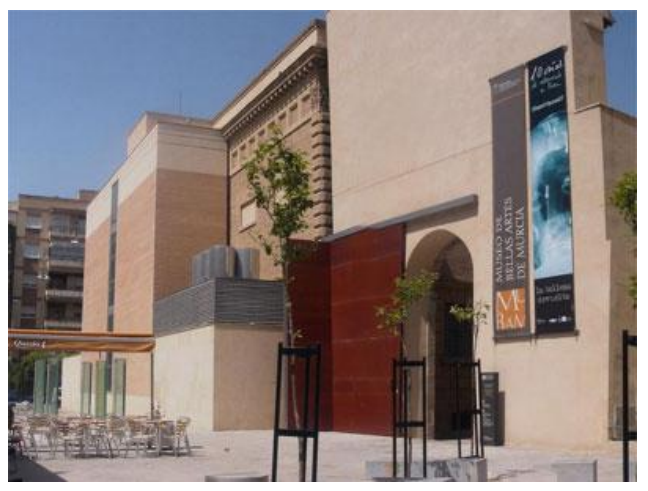

Ilustración 1: Museo de Bellas Artes de Murcia/ https://www.murciaturistica.es/es/museo/museo-de-bellas-artesde-murcia-46/ 
Tal y como se desarrollará a continuación, la colaboración horizontal entre profesionales del sector cultural y educativo, es una de las claves que permitirá que progresivamente los museos se transformen en espacios abiertos, a partir de proyectos e iniciativas que potencien la educación y el desarrollo sociocultural de la ciudadanía.

Los museos son espacios para la educación no formal, siendo este tipo de formación tan importante como la procedente de currículos reglados. De hecho, las posibilidades de trabajar de forma cooperativa, abre infinitas posibilidades al sector educativo. Si bien es cierto, que los departamentos educativos de los grandes museos, se han transformado en los últimos años, ofreciendo actividades para gran diversidad de colectivos, las instituciones más modestas no acaban de dar prioridad a este ámbito y si lo hacen, lo llevan a cabo desde una perspectiva tradicional, en la que solo tienen cabida los talleres plásticos para escolares. Sin embargo, la educación no formal que puede ofrecer un espacio museístico, puede ir mucho más allá, brindando la posibilidad de trabajar con aspectos relacionados con cuestiones contemporáneas como el género o la multiculturalidad.

Son diversos los museos que sí han percibido la gran perspectiva que permite aunar educación no formal con recursos culturales, y una de las claves de estos espacios suele ser la colaboración y la participación de los ciudadanos en la concepción de determinados proyectos o iniciativas.

El caso analizado del Museo de Bellas Artes de Murcia, es uno de los ejemplos de las infinitas opciones que un museo de tipo local, puede ofrecer para aportar al desarrollo de su comunidad. Para ello será necesario, dejar a un lado tendencias conservadoras que no favorecen el acceso de los individuos a las instituciones de forma participativa. Será preciso entonces crear estrategias que favorezcan ese flujo dinámico de ciudadanos hacia y desde la institución museística

\section{Marco Teórico}

En la Declaración de Córdoba, surgida a partir de la XVIII Conferencia Internacional de MINOM, celebrada en Córdoba (Argentina) en 2017, se recoge la siguiente frase (Bartolomé, Olga et al. (2019, p.125):

\section{La museología que no sirve para la vida, no sirve para nada.}

La denominada Museología Social o Sociomuseología, surge a partir de la publicación del primer número de los Cadernos de Sociomuseologia (1993), por Fernando Santos Neves, rector de la Universidade Lusófona de Humanidades e Tecnologías de Lisboa (Stoffel, 2012) y gira en torno a vincular la actividad de los museos al desarrollo de una determinada comunidad, tal y como expresa la cita anterior. 
Los museos son instituciones culturales al servicio de la ciudadanía, pero habitualmente se ha entendido esta premisa desde un punto de vista en el que la salvaguarda del patrimonio artístico era prioritario, tanto en lo relacionado con la distribución de recursos económicos, como en las actividades vinculadas al mismo. Sin embargo, las instituciones de este tipo tienen una responsabilidad social para con sus comunidades, ya que no sólo deben proteger nuestro legado cultural, sino también transmitirlo y difundirlo a toda la población.

Por tal razón, la evolución de la educación artística en las instituciones museísticas ha sido muy relevante a inicios del siglo XXI, llegando a un apogeo destacable en torno al año 2000 (Acaso, 2007). Sin embargo, en la última década, las cuestiones económicas que han afectado a la sociedad, han provocado que este importante avance retrocediera, llegando incluso a conocer la desaparición de algunos departamentos educativos.

En estos momentos, la educación en museos debe hacer frente a cuestiones sociales, siendo sensible a las inquietudes y problemáticas reales de la ciudadanía. Como ya indicaba Valdés (1999), gran número de museos, especialmente de tipo local, plantean unas actividades que nada o poco tienen que ver con el entorno que le rodea, lo que se traslada en la permanencia de ese concepto de museo como espacio inaccesible que predomina en una parte importante de la población.

En relación a esta llamada al acercamiento con la sociedad surge el perfil de mediador cultural, una figura que se acerca al ámbito de la educación informal y la animación sociocultural, ya que supone un avance en la implicación con el público. AMECUM ${ }^{1}$, apuesta por definir a los trabajadores de la educación en museos con este término:

AMECUM emplea habitualmente término "mediación" porque apunta a un campo más amplio en el que confluyen y dialogan diferentes agentes sociales y culturales cuya práctica profesional se basa en la producción de conocimiento de forma más abierta y participativa, siempre en diálogo con los públicos y usuarios (Asociación AMECUM, 2016).

Y es precisamente esa relación con los agentes sociales, culturales y educativos la clave para el avance hacia el concepto de museo social. Esa colaboración abierta a todo tipo de entidades o colectivos, permitirá que el museo conciba proyectos multidisciplinares que tengan como base la educación no formal, el arte y el desarrollo sociocultural.

No obstante, para que esta relación sea fructífera, se debe incidir en la forma de conectar tanto con la ciudadanía como con esos agentes y representantes de entidades socioculturales. La comunicación hoy en día es fundamental para cualquier organización de carácter público, y las nuevas tecnologías junto con la influencia de las redes sociales han provocado un cambio sustancial en las formas de difundir información e interactuar con el ciudadano.

\footnotetext{
${ }^{1}$ Asociación de Medidores Culturales de Madrid, http://amecum.es/
} 
Los grandes museos han sido conscientes de esta cuestión desde el inicio, pero no ha ocurrido por igual en otras instituciones más modestas, cuyo presupuesto no es destinado como prioridad a labores de marketing y comunicación digital.

En determinadas ocasiones, sigue existiendo desconocimiento sobre el verdadero objetivo de destinar recursos al aspecto comunicativo, ya que se confunde con un fin mercantilista. Sin embargo, si se consigue atraer a más público y por tanto obtener más recursos económicos, el beneficio recaerá sobre el propio museo, tal y como explica Li (2020, p.217)

La realización de las actividades de marketing en los museos no debe perseguir la obtención de beneficios económicos directos a corto plazo, sino convertirse en una estrategia conducente a que la imagen del museo cale en la población y provoque interés por visitarlo. (...) El objetivo fundamental de generar ingresos para los museos es más para obtener un beneficio social y el beneficio económico es solo un medio necesario para obtener ese beneficio social. (Li, 2020, p.217)

Las estrategias que apuesten por incentivar la presencia digital de los museos en la red, no deben verse como un aspecto negativo que trivialice el valor del patrimonio artístico de los museos, sino que es preciso visualizarlo como una vía fundamental para alcanzar la conexión con gran diversidad de públicos. Los recursos digitales no solo permiten la comunicación, sino que favorecen la difusión de conocimiento de infinitas maneras:

Lo virtual no es más que un sucedáneo de lo físico, pero benditos los sucedáneos digitales que permiten disfrutar de una exposición temporal que hace años cerró, expulsando de la ecuación al adjetivo temporal: las muestras virtuales no tienen fecha de caducidad (Mateos Rusillo, 2020).

No obstante, trabajar con el entorno digital no será suficiente por sí mismo para llegar al concepto de museo social, sino que deberá ser parte de una transformación progresiva del modus operandi de aquellas instituciones que siguen ancladas en el pasado. Colaboración, participación ciudadana, trabajo en red y acercamiento a las nuevas estrategias comunicativas serán las claves para encaminarse hacia el museo del futuro.

La investigación que se resume en este texto, trata de plantear un acercamiento a las nuevas metodologías que los museos deberán considerar para trabajar conjuntamente con su comunidad local. 


\section{Metodología}

El objetivo de esta investigación fue proponer una metodología adaptada a un medio específico con el fin de conseguir mejorar la relación entre el MUBAM y su contexto próximo. Por tal razón la participación de los ciudadanos y profesionales del entorno fue clave en el desarrollo de la investigación. Debido precisamente a la diversidad de perspectivas a afrontar para analizar la realidad llevada a estudio, se decide diseñar un método de investigación mixto, en el que se combinan diferentes técnicas como el cuestionario cuantitativo, la entrevista semi estructurada o el grupo de discusión.

El desarrollo del estudio fue dividido en varias fases donde se combinaron los distintos instrumentos de recogida de datos:

En una primera fase se suministraron los cuestionarios a los usuarios de los espacios para la cultura, la educación no formal y el desarrollo social, colaboradoras del estudio y presentes en los barrios colindantes del museo. Los centros y entidades participantes del estudio fueron entre otras, el Centro Cultural de La Paz, la Fundación Rais, Proyecto Hombre, Fundación Secretariado Gitano o la Asociación de Vecinos de Vistabella. En total participarían 19 organismos con un total de 426 cuestionarios respondidos, que facilitarían información sobre aspectos relevantes cómo el tipo de gestión de las propuestas socioculturales del entorno o bien las líneas de actuación vinculadas al desarrollo comunitario de la zona. Paralelamente a esta recogida de cuestionarios, se llevaron a cabo 22 entrevistas semi estructuradas. En las mismas participaron los técnicos o coordinadores de los 19 espacios socioculturales, junto con los 3 técnicos del Museo de Bellas Artes. Esta información unida a los datos obtenidos de los usuarios, aportó una perspectiva complementaria al estudio, sobre la relación entre entorno y museo.

En una segunda fase y tras analizar los datos mencionados, se llevó a cabo un grupo de discusión en el que participaron una selección de ciudadanos usuarios de los espacios socioculturales junto con los técnicos del MUBAM. En este encuentro se plantearon los resultados de la primera fase con el objetivo de proponer las líneas de trabajo a acometer por todos los implicados.

En una última etapa, se realizó una triangulación de todos los datos obtenidos empleando para ello la técnica del DAFO. Este recurso tendría como objetivo conocer tanto las debilidades y amenazas detectadas por parte de museo y entorno para convertir a la institución museística en un espacio colaborador del desarrollo comunitario, como las fortalezas y oportunidades para llevarlo a cabo, por parte de los propios ciudadanos y de la gestión del museo. Las distintas técnicas de regogida y análisis de datos, proporcionaron una perspectiva plural y heterogénea que daría como resultado una propuesta metodológica adaptada a un entorno concreto obtenida a partir de la participación de los propios implicados en la problemática. 


\section{Resultados}

La inclusión de los ciudadanos como parte fundamental del estudio propició que surgieran diferentes propuestas a ser consideradas tanto por el propio museo como por el resto de ciudadanos del entorno.

En relación a los cuestionarios completados por los usuarios habituales de los centros sociales, culturales y /o asociaciones del contexto cercano al museo, se obtienen interesantes conclusiones como, el reclamo de estos ciudadanos hacia una transformación en la forma de acceso del museo a los distintos colectivos sociales, que se percibe insuficiente. Por otro lado, al estudiar los datos se observa que existe una diferencia sustancial entre el tipo de actividades (y la forma de gestionarlas y difundirlas) del museo y el resto de espacios socioculturales, lo que se traduce en una relación más cómplice entre los técnicos y participantes de estos últimos organismos.

Se llevan a cabo 22 entrevistas, 19 a técnicos del entorno y 3 a técnicos del museo. Las respuestas a las preguntas formuladas (por igual a todos los entrevistados) exponen una serie de cuestiones relevantes, que permiten observar la diferencia de perspectivas entre institución museística y entorno.

Las opiniones por parte de los trabajadores del museo, demuestran que el museo sigue apostando por una gestión tradicional en la que la conservación y exposición del patrimonio artístico es una prioridad que ocupa gran parte de la actividad del mismo. La relación con la educación se suele limitar a los talleres didácticos para escolares, ejecutados también desde una metodología clásica. No obstante, el museo es consciente de estas limitaciones y se manifiesta unánime a favor de crear nuevas líneas de trabajo educativas no formales para abarcar a otros colectivos sociales.

Por otro lado, la información recopilada por los coordinadores y técnicos de las asociaciones del entorno denota una sensación generalizada de crear estrategias que optimicen la relación del museo con el entorno, haciéndolo más accesible para la comunidad. Se sigue considerando al museo como un espacio conservador de carácter cerrado y poco sensible a la realidad contextual. Los participantes reclaman una vinculación más estrecha y colaborativa con la institución museo, para poder integrar a todos los colectivos sociales presentes en la comunidad mediante actuaciones de divulgación y educación del patrimonio artístico. 
Los datos anteriores sirvieron para diseñar las líneas de debate a consultar en el grupo de discusión. Del tal encuentro se obtienen propuestas formuladas tanto por los ciudadanos, como por los técnicos del entorno o del museo.

La mejora en las estrategias de comunicación entre el museo y los distintos centros y entidades socioculturales y educativas del contexto, adaptándose a la gran diversidad de públicos existentes o la realización de proyectos y actuaciones conjuntas entre ambos contextos, serían las ideas más reclamadas durante el encuentro. En esta discusión todos los participantes coincidieron en la necesidad de facilitar este tipo de iniciativas en las que se conocen de primera mano las inquietudes y necesidades reales de la ciudadanía.

Las actuaciones planteadas en la sesión de debate, servirían de base para elaborar la propuesta metodológica para optimizar la colaboración entre museo y entorno a partir de la participación de los ciudadanos.

El DAFO empleado nos permite analizar todos los datos, concluyendo con la idea de que serían siete las áreas de actuación en las que trabajar: la museología, la gestión, las infraestructuras, la educación y acción cultural, la difusión y comunicación, las estrategias de colaboración y los estudios de público.

Entre las iniciativas resultantes, destacarían las vinculadas a la difusión o a la educación no formal, tales como la revisión y adecuación de las líneas de comunicación entre museo y ciudadanía adaptándose a las nuevas realidades sociales, o bien la realización de proyectos socioeducativos en colaboración entre museo y demás entidades participantes.

Se pudo constatar, por tanto, la necesidad de vincular los museos al resto de la sociedad, pero no solo con actividades dirigidas desde la institución a diversos públicos, sino a través de la inmersión de agentes de las distintas organizaciones y entidades socioculturales que componen nuestra sociedad. Las instituciones museísticas deben actuar como espacios mediadores, empleando el arte como recurso para educar a los ciudadanos.

\section{Líneas de trabajo futuras: contextos colaborativos}

Tras un año del término de esta investigación y después de una revisión de la misma a teniendo en cuenta el momento actual, se plantean dos nuevas vías de trabajo relacionadas también con la experiencia profesional como docente en el Departamento de Didáctica de la Expresión Plástica de la Facultad de Educación de la Universidad de Murcia 
La primera vía de trabajo sería la vinculada a una de las grandes demandas detectadas en la investigación inicial: las diferentes vías de mediación y comunicación entre museo y colectivos sociales, especialmente las relacionadas con las nuevas tecnologías participativas. Esta última además se vería influida por los acontecimientos de este año 2020, ligados a la pandemia del COVID 19 y sobre cómo los museos se han enfrentado a esta situación.

Una segunda línea de investigación trabajaría en torno a la vinculación entre el contexto universitario (formación de profesorado) y las instituciones museísticas.

\subsection{Museo /entorno sociocultural}

La primera línea con la que seguir trabajando es la vinculada a una de las demandas más relevantes detectadas durante la recogida de datos y posterior análisis: la necesaria mejora en las estrategias de comunicación entre museo y entorno. El museo cuenta como medio principal de comunicación con una newsletter que envía periódicamente a los ciudadanos que previamente se han registrado. Además, su presencia en redes sociales en el momento de la investigación era completamente nula, hecho que ha cambiado, pero no sustancialmente, ya que en estos momentos tan solo existe una página (no muy activa) del conjunto de museos gestionados por la región. Al no contar con un perfil propio en redes, la dinámica comunicativa con los ciudadanos es muy limitada.

Tras el grupo de discusión realizado, las propuestas que surgen en torno a cómo optimizar este sistema de comunicación y difusión, tanto por parte tanto de ciudadanos, técnicos del entorno como profesionales del museo, son las siguientes (Castejón, 2019, p.347):

- $\quad 19 \%$ Incluir en la base de datos de los correos electrónicos que envía el museo a los centros y asociaciones.

- $14,2 \%$ Aumentar presencia en prensa, radio y tv

- $14,2 \%$ Más presencia en las redes sociales

- $\quad 19 \%$ Incluir en la base de datos de los correos electrónicos que envía el museo a los centros y asociaciones

- $\quad 14,2 \%$ Recuperar la difusión en papel (cartelería, folletos...)

- $\quad 9,5 \%$ Buscar medios de comunicación alternativos para los centros que no tienen correo electrónico

- $\quad 9,5 \%$ Darse a conocer en los centros y asociaciones cercanas al museo

- $\quad 4,7 \%$ Fomentar el boca a boca

- $\quad 4,7 \%$ Mejorar difusión de exposiciones temporales

- $\quad 9,5 \%$ Publicar la programación de modo bimestral o trimestral

Según lo observado, la gran mayoría de opiniones giran en relación a mejorar la visibilidad del museo a diversos colectivos, haciendo hincapié en la necesidad de ganar presencia en las redes sociales. 
Esta reivindicación toma un mayor sentido en el momento que nos encontramos con la crisis generada por la pandemia mundial del COVID-19. Los museos, como el resto de la sociedad, se han tenido que adaptar a las nuevas exigencias médicas que demandan distancia social o grupos muy reducidos de público. Tal es el caso de los museos de la Región de Murcia, donde estos meses se ha percibido una notable bajada de visitantes (Hernández, 2020).

Las redes sociales han sido un recurso muy útil empleado por muchos museos para seguir conectados con sus públicos, como es el caso del Museo del Prado o del Museo Reina Sofía (Gallardo, 2020). Si bien es cierto que estas instituciones cuentan con mayores facilidades para afrontar estas vicisitudes, los museos con menores posibilidades deben apostar por actualizar sus estrategias para poder llegar a más colectivos sociales. Tal es el caso del MUBAM, cuya presencia virtual durante el espacio temporal que la institución estuvo cerrada (marzo/junio de 2020), fue prácticamente nula.

La inmersión en la tecnología por parte de los museos debe pensarse como una vía de acercamiento a la sociedad plural actual. De hecho, la situación originada por la pandemia mundial, debería provocar ese necesario replanteamiento de la gestión de gran número de museos, caminando hacia una visión que apueste por la sostenibilidad y lo social (Mateos Rusillo, 2020). Es precisamente en este sentido hacia donde se propone esta línea de trabajo: seguir desarrollando el concepto de museo social iniciado con la investigación citada, llegando a encontrar un equilibrio entre las nuevas estrategias comunicativas y el desarrollo sociocultural de los ciudadanos.

\subsection{Museo /Universidad}

En la investigación realizada no participó ninguna asociación ni representación del ámbito de la educación formal, tales como colegios, institutos o universidades. Esta decisión estuvo condicionada por los recursos materiales y temporales de dicho estudio y la imposibilidad de abarcarlo. No obstante, la relación entre colegios y museos suele estar establecida, ya que los talleres escolares son las actividades más habituales en instituciones museísticas como es el caso del MUBAM.

En la primera etapa de la investigación, y en concreto durante las entrevistas a los técnicos del museo, queda patente que uno de los sectores de público habituales en la institución son los profesionales del arte (10\%) o estudiantes del sector (20\% universitarios), ya sean historiadores, artistas o gestores culturales. Sin embargo, en ningún momento se menciona la participación del sector del profesorado o de estudiantes del ámbito educativo, es decir, de los profesionales que tanto en el presente como en un futuro cercano serán quienes organicen esas visitas didácticas a los espacios museísticos.

Estos datos iniciales, se unen a la experiencia observada durante el desarrollo del curso 2019/2020 en una asignatura del Grado de Educación Primaria, vinculada al ámbito artístico. 
ISSN: $2340-9096$

https://dx.doi.org/10.17561/rtc.extra3.5715

Al proponer a los alumnos la creación de una actividad didáctica que tuviera como escenario un museo de arte contemporáneo, se observa que un gran porcentaje de alumnos desconoce las posibilidades que ofrece un museo como recurso complementario al currículo formal. Al proponerles investigar sobre la institución museística, la mayoría se sorprende al descubrir la existencia de un departamento educativo en el propio museo, así como instalaciones para llevar a cabo las actividades didácticas y/o artísticas.

Durante la exposición de los trabajos en el aula, muchos de los alumnos coinciden en lo idóneo de considerar estos espacios como oportunidades para trabajar aspectos relevantes como las cuestiones de género, la identidad cultural o las problemáticas del medio ambiente, que van más allá de la mera experiencia plástica. Esta observación previa dará pie a plantear un estudio posterior (en desarrollo), en el que se podrá valorar más concretamente la relación entre la formación del futuro profesorado y los museos. No cabe duda que una de las principales cuestiones a tratar será la relación que tienen en la actualidad estos futuros docentes con las instituciones, ya que tal y como ya avanzó Aguirre (2013, p.9) en un estudio similar (llevó a cabo una encuesta a estudiantes de educación infantil), la situación no es muy positiva:

(...) la gran mayoría de las estudiantes preguntadas afirman que en la actualidad no visitan nunca museos ni centros de arte o salas de exposiciones.

Los próximos maestros y maestras deberán asumir esa responsabilidad como mediadores entre la educación y la cultura, recurriendo para ello entre otras estrategias a la colaboración estrecha con los educadores de museos. 


\section{Conclusiones}

A través de este texto se han presentado los resultados de la investigación realizada entre el Museo de Bellas Artes de Murcia y su entorno sociocultural cercano.

Entre las principales conclusiones detectadas se encuentra la necesidad de mejorar el sistema de comunicación con los agentes del contexto. Tanto los ciudadanos, como los técnicos del museo y los agentes del entorno, coinciden en destacar la importancia de establecer estrategias para optimizar el encuentro entre ciudadanos y museo, de lo que surgen diversas propuestas, centradas principalmente en dos aspectos: la comunicación y la colaboración. A partir de dichas propuestas y con el objetivo de revisar y actualizar el estudio, se plantean dos líneas de trabajo futuras a desarrollar que se exponen en este texto. La relación más estrecha con la universidad será determinante para cambiar desde la base la forma de relacionar los contextos educativos formal y no formal. Para ello se propone diseñar un nuevo estudio que analice la vinculación del futuro profesorado con las instituciones museísticas. Este tendrá como objetivo mejorar desde la universidad la formación de estos futuros docentes con respecto a las posibilidades de los museos como complemento a los currículos oficiales.

Por otro lado, se propone un acercamiento a los nuevos modos de comunicación ligados a la tecnología y las redes sociales. Los museos se van acercando progresivamente a este tipo de estrategias que favorecen la conexión con gran amplitud de público, sin embargo, muchos son los que no consideran estos medios como prioritarios dentro de su gestión.

La reciente situación de crisis provocada por la pandemia mundial, cuyo fin no se percibe aún en el horizonte, debe provocar a los museos, como ha ocurrido con otros sectores, que replanteen sus metodologías. Para ello será necesario, apoyarse en las opciones que las nuevas tecnologías permiten, sin olvidar que el objetivo de los museos del futuro será transformarse en espacios clave en el desarrollo sociocultural de su entorno.

La colaboración entre agentes, a partir de la mejora en las estrategias comunicativas, será clave para conseguir que los museos sean instituciones fundamentales para la educación no formal, basada en los recursos artísticos que atesoran. 
ISSN: $2340-9096$

https://dx.doi.org/10.17561/rtc.extra3.5715

\section{Referencias.}

Acaso, M. (2007). ¿Por qué a la educación artística no le gustan los museos? Repensando los problemas actuales de la educación artística en las instituciones culturales. En Calaf Masachs, R., Fontal Merillas, O. y Valle Flórez, R. E. (Coord.), Museos de arte y educación. Construir patrimonios desde la diversidad (pp.75-82) Gijón: Trea.

Acaso, M. y Megías, C. (2017). Art Thinking. Cómo el arte puede transformar la educación. Madrid: Paidós Educación.

Aguirre Arriaga, Imanol. (2013). El papel de la educación en el acceso democrático a la cultura y las artes. Encuentros y desencuentros entre escuela y museo. Revista pensamiento, palabra y Obra, (10). https://doi.org/10.17227/ppo.num10-2120

Bartolomé, Olga et al. (2019). Dossier: Nueva Museología, Museología Social. Revista Del $\begin{array}{lllll}\text { Museo De } & \text { AntropologíA, } & \text { 12(2), } & \text { 128. }\end{array}$ doi:http://dx.doi.org/10.31048/1852.4826.v12.n2.25236

Castejón, M. (2019). Hacia un museo social: estudio de una metodología para vincular el Museo de Bellas Artes de Murcia a su territorio. Tesis doctoral. Universidad de Murcia. Disponible en: http://hdl.handle.net/10201/73564

Li, Yin. (2020). Museos y Marketing: una controversia ante nuevas estrategias, Esic Market Economics and Business Journal, 51(1), 209-234. Doi: 10.7200/esicm.165.0511.4

Gallardo, Vicky. (8-6-2020). La nueva era de los museos: del comisario al community manager. El Mundo. Recuperado de: https://www.elmundo.es/cultura/2020/06/08/5ecf88f2fc6c83c3408b45e4.html

Hernández, Esperanza (21-08-2020). Los museos de la Región de Murcia, ¿afectados por la covid?, Murciaplaza, Recuperado de, https://murciaplaza.com/los-museos-de-la-regionde-murcia-afectados-por-la-covid

Mateos Rusillo, Santos (17-05-2020). Tras la pandemia: ¿museos más justos, sostenibles y sociales? The Conversation, Recuperado de: https://theconversation.com/tras-lapandemia-museos-mas-justos-sostenibles-y-sociales-137123

Stoffel, Ana Mercedes. (2012). De que hablamos cuando hablamos de Sociomuseología. En $\begin{array}{llll}\text { RdM.Revista de } & \text { Museología, } & \text { (53), } & \text { pp. }\end{array}$ 